\title{
Observation of a kink during the formation of the Kondo resonance band in a heavy-fermion system
}

\author{
Hong Chul Choi, ${ }^{1,2}$ K. Haule, ${ }^{3}$ G. Kotliar, ${ }^{3}$ B. I. Min,,${ }^{1, *}$ and J. H. Shim ${ }^{1,2,4, \dagger}$ \\ ${ }^{1}$ Department of Physics, Pohang University of Science and Technology, Pohang 790-784, Korea \\ ${ }^{2}$ Department of Chemistry, Pohang University of Science and Technology, Pohang 790-784, Korea \\ ${ }^{3}$ Department of Physics, Rutgers University, Piscataway, New Jersey 08854, USA \\ ${ }^{4}$ Division of Advanced Nuclear Engineering, Pohang University of Science and Technology, Pohang 790-784, Korea
}

(Received 13 February 2013; revised manuscript received 19 August 2013; published 6 September 2013)

\begin{abstract}
We have shown that the kink behavior in the spectral function of a heavy fermion can appear during the formation of the Kondo resonance (KR) band and the hybridization gap. We have investigated the heavy fermion compound $\mathrm{CeCoGe}_{2}$, using a combined approach of the density functional theory and the dynamical mean field theory. Low temperature $T$ spectral functions show dispersive KR states, similarly to the recent experimental observation. During the evolution from the non- $f$ conduction band state at high $T$ to the dispersive KR band state at low $T$, which have topologically different band shapes, we have found the existence of kinks in the non- $f$ spectral function near the Fermi level $E_{F}$. The observation of kink is clearly in correspondence with the multiple temperature scales of the formation of the KR band.
\end{abstract}

DOI: 10.1103/PhysRevB.88.125111

PACS number(s): 71.20.-b, 71.27.+a, 75.30.Mb

The Hamiltonians for the heavy fermion compounds, such as the Kondo lattice model and the periodic Anderson lattice model, ${ }^{1}$ are represented in terms of the kinetic energies of conduction electrons, the correlation energies among localized electrons, and the hybridization between them. In Ce-based compounds, the localized electrons and the conduction electrons correspond to Ce $4 f$ electrons, and other spd electrons. The non- $f$ electrons are mostly metallic (delocalized) and well described by the density functional theory (DFT). The localization of $4 f$ electrons in the ground state is determined by the competition between the Ruderman-Kittel-KasuyaYosida (RKKY) interaction and Kondo effect. ${ }^{1}$ The RKKY interaction is the conduction electron-mediated exchange energy between $4 f$ electrons. The Kondo effect induces the spin singlet state of the localized and delocaled electrons. The hybridization strength between $4 f$ and non- $f$ electrons strongly affects both the RKKY interaction and the Kondo effect. At the weak hybridization, the RKKY interaction drives the localized $4 f$ electrons to be magnetic. The Kondo effect at the strong hybridization produces the Fermi liquid states with heavy mass.

Depending on the temperature, the localization of $4 f$ electrons are changed in heavy fermion compounds; at high temperature $T$, the $4 f$ states are well localized to have a negligible contribution to the Fermi level $E_{F}$, and so only non- $f$ dispersive conduction bands are observed near $E_{F}$ with effectively small hybridization. At low $T$, however, the Kondo effect overwhelms the RKKY interaction, and the $4 f$ states appear near $E_{F}$ and to be hybridized with other conduction electrons. Due to the flatness of the hybridized bands, the effective mass of quasiparticles becomes tens to hundreds times larger than the bare electron mass. This quasiparticle band near $E_{F}$ can be identified as the dispersive Kondo resonance (KR) peak $^{2}$ in the angleresolved photoemission spectrum (ARPES). ${ }^{3,4}$ The upper and lower hybridized bands produce the hybridization gap ${ }^{1}$ (HG), which can be recognized below the coherent temperature $T^{*}$, when the heavy quasiparticle bands begin to emerge.
The HG feature was directly observed by the optical conductivity, ${ }^{5-10}$ and described theoretically by the periodic Anderson model. ${ }^{1}$ The optical conductivities show the midinfrared peaks in the energy range of several tens of meV at low $T$. These peaks are suppressed with increasing $T$ and disappear well above $T^{*}$ to produce the incoherent Drude peak at zero frequency. The magnitude of the $\mathrm{HG}\left(\Delta_{H G}\right)$ measures the hybridization strength between $f$ and other non- $f$ conduction states. Recently, the dynamical mean field theory (DMFT) approach revealed the formation of the $\mathrm{HG}$ in $\mathrm{CeIrIn}_{5}$ by calculating the $T$-dependent spectral function $A(k, \omega) .{ }^{11}$ The momentum-dependent $\Delta_{H G}$ at low $T$ was in good agreement with the measured spectrum and provided the information of the orbital-dependent hybridization strength.

In this study, we have theoretically described the formation of the dispersive KR band and the $\mathrm{HG}$ in the heavy fermion compound $\mathrm{CeCoGe}_{2} \cdot{ }^{12}$ It has the orthorhombic base-centered structure, shown in Fig. 1. Though the nearest neighbors of $\mathrm{Ce}$ atoms are $\mathrm{Ge}$ atoms, the $s p$ states in Ge ions are almost empty. The open-core calculation ( $4 f$ states set to be inside the core), which is equivalent to the electronic structure at high $T$, demonstrated that Co $3 d$ states have the most contribution to $E_{F}$. So, Co $3 d$ states can be regarded as the main character of non- $f$ states. The KR states are composed of dispersive bands arising from the hybridization of non- $f$ and renormalized $f$ bands, and show quantitative agreement with the recent ARPES measurement. ${ }^{3}$ We have shown that the formation of the KR band, which brings about a topological change of the band structures, should be accompanied by the kinks in the spectral function. We propose that the kink could appear in the non- $f$ spectral function $\left[A^{\text {non- } f}(\mathbf{k}, \omega)\right]$ prior to the formation of the KR band around $T^{*}$.

The kink, the abrupt change in the band dispersion, is usually observed in the ARPES measurements of high $T_{C}$ superconductors. ${ }^{13-17}$ The discontinuous quasiparticle band is induced by various collective excitations, such as phonon ${ }^{13-15}$ and spin fluctuation. ${ }^{16}$ When the strong electron-phonon coupling disturbs the velocity and the scattering rate of electrons, ARPES spectrum ${ }^{18-20}$ near the phonon energy 
(a)

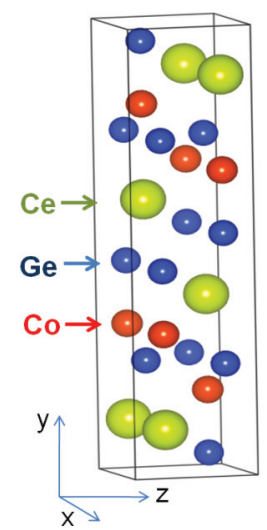

(b)

Conventional Brillouin zone

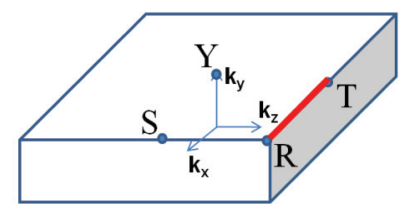

FIG. 1. (Color online) Crystal structure and its Brillouin zone (a) The crystal structure of $\mathrm{CeCoGe}_{2}$ (Ref. 33). (b) The conventional Brillouin zone. Red line represents the path used in Fig. 3.

shows the abrupt change in the slope of the energy-momentum dispersion. The ARPES experiment on $\mathrm{USb}_{2}$ also revealed the kink feature in the dispersion of $f$ bands, which was explained by the combination of quasiparticle bands and the many-body correction of the electron-spin-fluctuation coupling. ${ }^{21}$ On the other hand, it was reported that the pure electronic correlation in the Hubbard model can produce such a kink near $E_{F},{ }^{22}$ which suggests that the one-particle picture should be strongly renormalized below a certain energy $\omega^{*}$ to have the kink feature in momentum space. This analogy was applied to the periodic Anderson model, showing the existence of the kink. ${ }^{23}$ Recently, the energy scale of the kink was proved to be smaller than the width of the central peak. ${ }^{24}$ Other subsequent studies, however, showed that the kink in the Hubbard model arises from the internal spin-fluctuation mode. ${ }^{25-27}$ In the present study, instead of kinks in correlated bands, we show that kinks can be phenomenologically observed in noncorrelated bands due to the formation of correlated $f$ states in the heavy fermion system.

We have used the DFT + DMFT method ${ }^{28}$ implemented in the linearized muffin-tin orbital band method. ${ }^{29}$ We have considered the experimental crystal structure and the Brillouin zone shown in Fig. 1. The DMFT calculation considers only the local self-energy of the $4 f$ orbital, and other orbitals are considered in the DFT part. The local correlation effect is contained in the self-energy $\Sigma(\omega)$, which can be calculated from the corresponding impurity problem. To solve the impurity problem, we used a vertex corrected one-crossing approximation (OCA), ${ }^{28}$ which is a self-consistent diagrammatic method perturbed in the atomic limit. ${ }^{30}$ We used the same interaction parameters $U=5.0$ and $J=0.8 \mathrm{eV}$, as in previous works on CeIrIn5. ${ }^{11,31}$ It has been checked that the OCA describes well the $T$-dependent spectral function of the heavy fermion compound. ${ }^{11,31}$ We neglected the crystalline electric field (CEF) effect on the local correlation energy because $\mathrm{CeCoGe}_{2}$ has been confirmed as a $j=5 / 2$ heavy fermion. ${ }^{12}$

Figures 2(a) and 2(b) show $A^{\text {non- } f}(\mathbf{k}, \omega)$ 's along $S-R-T-Y$ at $T=1200$ and $10 \mathrm{~K}$, which demonstrates the formation of the KR bands near $E_{F}$. The dispersive $s p d$ bands at high $T(1200 \mathrm{~K})$ look almost vertical due to the narrow energy range. At low $T$, as a result of the hybridization,


FIG. 2. (Color online) Theoretical demonstration of the HG feature from $T$-dependent $A^{\text {non- } f}(\mathbf{k}, \omega) . A^{\text {non- } f}(\mathbf{k}, \omega)$ 's are provided along $S-R-T-Y$ (a) at $T=1200$ and (b) at $T=10 \mathrm{~K}$. $S, R, T$, and $Y$ are $\mathbf{k}=(\pi / 2, \pi / 2,0),(\pi / 2, \pi / 2, \pi / 2),(0, \pi / 2, \pi / 2)$, and $(0, \pi / 2,0)$, respectively. The green circle in (b) is for the emphasis of the $T$-dependent change. Blue dotted lines marked by $A, B, C$, and $D$ represent $\mathbf{k}$ points, where the $T$-dependent $A^{\text {non- } f}(\mathbf{k}, \omega)$ calculations in (c) to (f) are done. Theoretically predicted $\Delta_{H G}$ is given at each $\mathbf{k}$ point.

unusual coherent quasiparticle bands are formed to give the different band geometry near $E_{F}$. For example, there are two separate bands crossing $E_{F}$ between $S$ and $R$ at high $T$, while at low $T$, additional $j=5 / 2$ bands with the bandwidth of $\sim 10 \mathrm{meV}$ are introduced to produce degenerated three composite quasiparticle bands. As a result, the lower part of two bands observed at high $T$ are warped due to the hybridization, as indicated by the green circle in Fig. 2(b). One band closer to $S$ is pushed down below $E_{F}$, but another band still intersects $E_{F}$. It is interesting that the formation of the paraboliclike hybridized band originated from high $T$ separated bands below $E_{F}$ around $k=D$ at low $T$. All those features result in the change of geometry in the Fermi surface (FS), as will be shown in Figs. 4(e) and 4(f).

The $T$-dependent formation of the HG has been examined at the chosen $\mathbf{k}$ points in Figs. 2(c) to 2(f). At high $T$, $A^{\text {non- } f}(\mathbf{k}, \omega)$ shows a general quasiparticle spectral feature with a single Gaussian function at each $k$ point. With lowering $T$, the spectral weights of $A^{\text {non- } f}(\mathbf{k}, \omega)$ near $E_{F}$ begin to be transferred to upper and lower peaks separated by $\Delta_{H G}$ to form a gap structure. At low $T$, the clear gap can be observed at each chosen $\mathbf{k}$ point. The size of gap $\Delta_{H G}$ in $A^{\text {non- } f}(\mathbf{k}, \omega)$ can be measured by the optical conductivity. Because $\Delta_{H G}$ has a variation of 70 to $100 \mathrm{meV}$ depending on the $\mathbf{k}$ points, the 

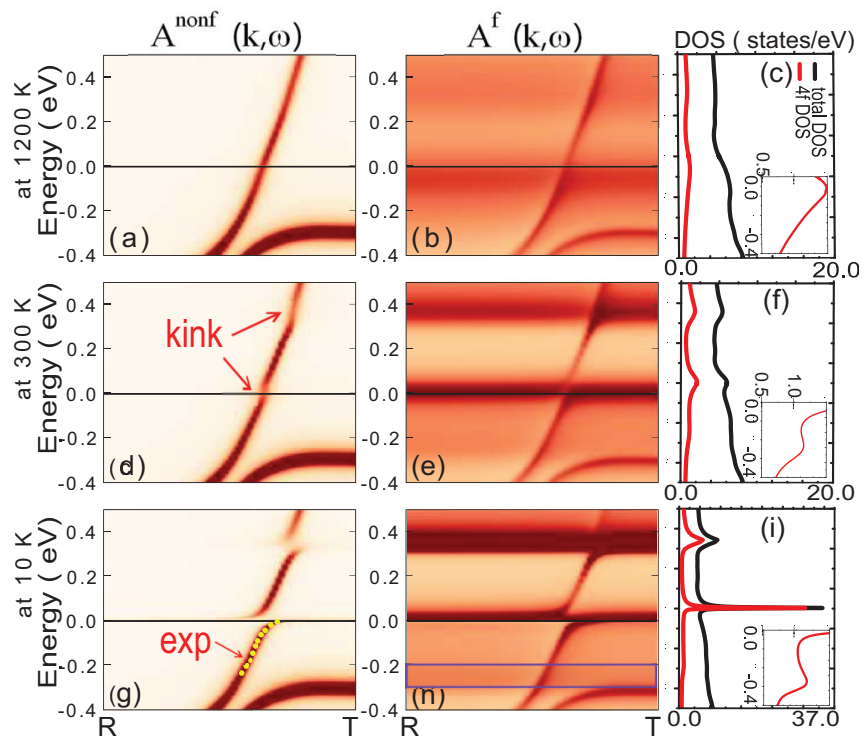

(i)

FIG. 3. (Color online) (a),(d),(g) $T$-dependent $A^{\text {non- } f}(\mathbf{k}, \omega)$ and (b),(e),(h) $A^{f}(\mathbf{k}, \omega)$ between $R(\pi / 2, \pi / 2, \pi / 2)$ and $T(0, \pi / 2, \pi / 2)$. The corresponding DOS's are also given in (c), (f), and (i). The insets show the detail of the spin-orbit multiplet at $-300 \mathrm{meV}$. The purple rectangle in (h) indicates the weak spectrum of the spin-orbit multiplet, which can be seen clearly at $-0.3 \mathrm{eV}$ below $E_{F}$ in (i). Yellow points in $(\mathrm{g})$ represent the off-resonant ARPES spectra in the measurement (Ref. 3), which are shifted along the $\mathbf{k}$ path for better alignment of non- $f$ bands.

measured $\Delta_{H G}$ should show multiplet structures or widespread shape in the optical conductivity measurements. The small peaks near $E_{F}$ at $20 \mathrm{~K}$ are induced due to the formation of quasiparticle bands of $j=5 / 2$ states within the HG.

Figure 3 shows both $A^{\text {non- } f}(\mathbf{k}, \omega)$ and the $f$ spectral function $\left[A^{f}(\mathbf{k}, \omega)\right]$ along $R-T$, and the integrated density of states (DOS) around $E_{F}$ at $T=1200,300$, and $10 \mathrm{~K}$. The $T$-dependent development of the $\mathrm{KR}$ states is clearly confirmed in the DOSs of Figs. 3(c), 3(f), and 3(i). At high $T$, the upper and lower Hubbard bands are located near 2 to $3 \mathrm{eV}$ above $E_{F}$ and $2 \mathrm{eV}$ below $E_{F}$, respectively (not shown here). At the elevated $T$, the profile of the DOS near $E_{F}$ comes mostly from non- $f$ states, although there is a weak background spectrum of $4 f$ states. With lowering $T$, the weights of the upper and lower Hubbard bands are reduced and transferred to the KR states near $E_{F}$. The $4 f$ states give the main contribution to the DOS near $E_{F}$ below $T^{*}$.

At high $T(1200 \mathrm{~K}), A^{f}(\mathbf{k}, \omega)$ shows weak intensity near $E_{F}$, but has a clear dispersive band feature similar to $A^{\text {non- } f}(\mathbf{k}, \omega)$. This means that small hybridization still exists between non- $f$ and $f$ states at high $T$. The broad Gaussian peaks in $A^{\text {non- } f}(\mathbf{k}, \omega)$ at high $T$, as shown in Figs. 2(c) to 2(f), are the indication of this hybridization. At low $T(10 \mathrm{~K})$, where the KR states are fully developed, both $A^{f}(\mathbf{k}, \omega)$ and $A^{\text {non- } f}(\mathbf{k}, \omega)$ show the KR band structures with different weight distributions. The non- $f$-dominant bands form the gap structure with low intensity at $E_{F}$, while the $f$-dominant bands are confined near $E_{F}$ to give the sharp KR peak in the integrated DOS, as shown in Fig. 3(i). Note that the KR states should be considered as dispersive band structures, as described in the periodic Anderson model.
$A^{\text {non- } f}(\mathbf{k}, \omega)$ and $A^{f}(\mathbf{k}, \omega)$ at low $T(10 \mathrm{~K})$ show good agreement with the off-resonance and on-resonance ARPES measurements at $T=17 \mathrm{~K},{ }^{3}$ respectively. Especially, the momentum dependence of dispersive KR peaks in the experiments is well consistent with the calculated spectrum, as shown in Fig. 3(g). Also the weight distribution of non- $f$ and $f$ states observed in experiments are correctly captured in the calculation. The weak dispersionless spin-orbit multiplet peak, which exists inside the purple rectangle in Fig. 3(h), is also consistent with the experimental observation. The spin-orbit multiplets of $A^{f}(\mathbf{k}, \omega)$ are shown around $\pm 0.3 \mathrm{eV}$, and their intensities increase as lowering $T$. Insets in Figs. 3(c), 3(f), and 3(i) provide the $T$-dependent enhancement of the spin-orbit multiplet around $-0.3 \mathrm{eV}$. It is noteworthy that the multiplet shows almost flat feature because the incoherent feature (broadening of bands) is much bigger than the dispersion of the KR states.

Because the spectra of high and low $T$ show clearly different quasiparticle band structures near $E_{F}$, the $T$-dependent evolution should show some feature of phase transition or crossover. Figures 3(d) and 3(e) show the spectra in the intermediate $T . A^{f}(\mathbf{k}, \omega)$ around $E_{F}$ shows an effectively dispersionless feature, which is the precursor of the formation of the KR states. Below and above the KR state, the non- $f$ bands are warped in different directions. At the energy of the KR state, the non- $f$ bands are not well defined due to the incoherent contribution of $A^{f}(\mathbf{k}, \omega)$ to the non- $f$ states. As a result, $A^{\text {non- } f}(\mathbf{k}, \omega)$ shows the feature of kinks near $E_{F}$. Distinctly from the kinks observed in other correlated systems, such as a high $T_{C}$ superconductor, the kinks in the heavy fermion system should appear in the noncorrelated bands during the formation of the KR bands and the HG.

Figures 4(a) to 4(d) show the schematic picture of emergence of kink during the formation of the KR bands. ${ }^{32} \mathrm{At}$ high $T$ in Fig. 4(a), there are only non- $f$ conduction bands that can be usually well described by the open-core band calculation, in which the occupied Ce $4 f$ state is treated as a core level. With lowering $T$ in Fig. 4(b), the incoherent KR states of $4 f$ electrons start to contribute to $E_{F}$, whereby the kink feature starts to emerge in $A^{\text {non- } f}(\mathbf{k}, \omega)$. Here the kink structure is far from the "water-fall" shape, rather close to a "bell-profile" shape, indicated by the arrows in Fig. 3(d), since the dispersion changes happen at two spin-orbit multiples ( $j=5 / 2,7 / 2$ ). As decreasing $T$ further, the $f$ electrons start to be coherent slowly, and the non- $f$ bands are still being deformed. This process corresponds to Fig. 4(c), where the coherent character of bands becomes enhanced around $E_{F}$. In this case, $A^{\text {non- } f}(\mathbf{k}, \omega)$ has the kink structure of the water-fall shape due to the separation of the upper and lower hybridized bands. At lower $T$ in Fig. $4(\mathrm{~d})$, most $4 f$ electrons near $E_{F}$ become coherent to make the fully coherent bands near $E_{F}$. Accordingly, the region of the kink feature is changed into that of the HG feature. Interestingly, the electron FS area gradually enlarges during this procedure.

The area of the electron FS around $R$, which is identified as the $\alpha$ branch in Figs. 4(e) and 4(f), increases continuously with lowering $T$. In our recent DMFT study ${ }^{31}$ on the FS of the heavy fermion CeIrIn 5 , two temperature scales are proposed: one ( $T_{f}$ ) for the $T$-dependent evolution of the FS size, and the other 
(a) High T

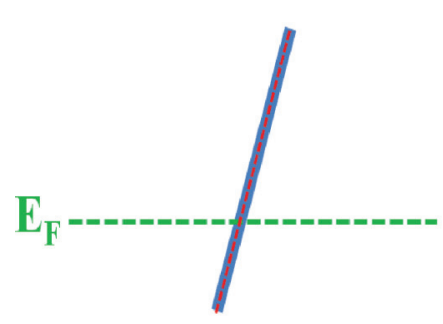

conduction band (b) Kink happens

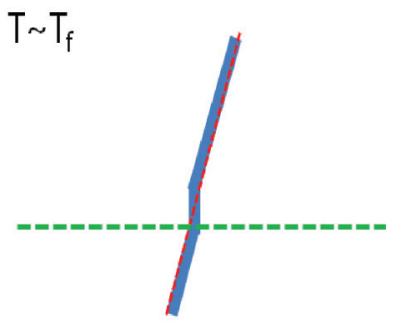

(f) LOW T FS

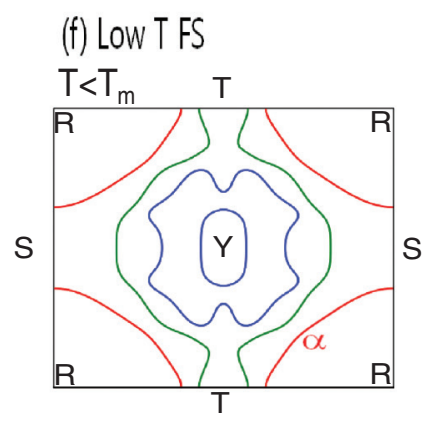

(c) Enhanced Hybridization

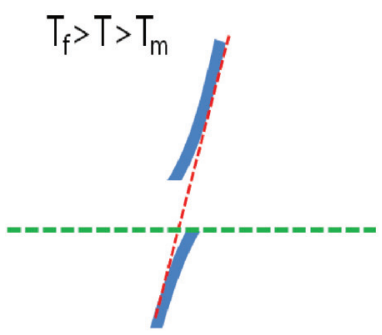

(g)

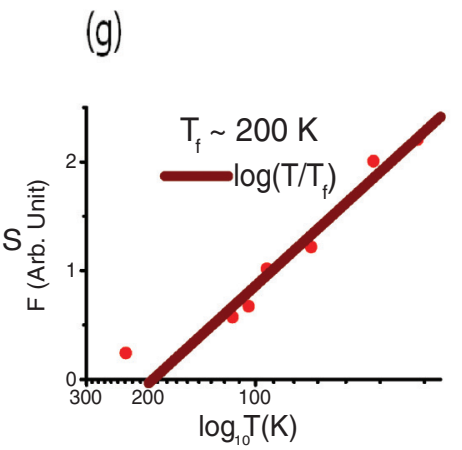

(d) After Hybridization

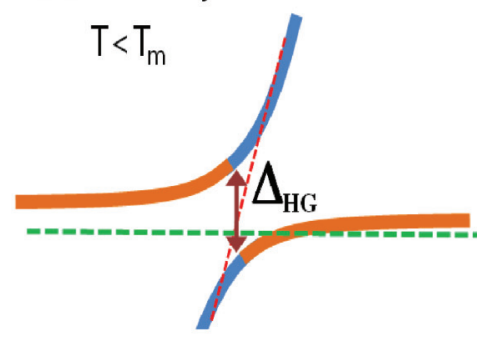

dispersive KR states



(h)

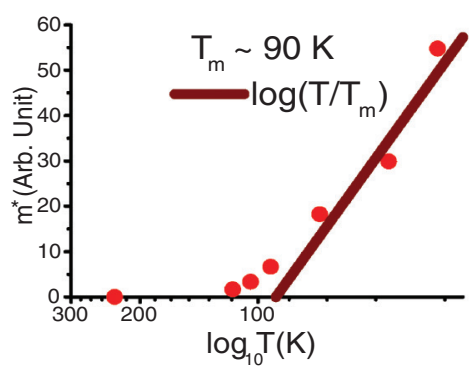

FIG. 4. (Color online) The formation of the HG can be divided into four processes in (a) to (d), using $T_{f}$ and $T_{m}$ that are obtained by the scaling analysis in (g) and (h). (a) The high $T$ quasiparticle band is displayed. The blue color represents $A^{\text {non- } f}(\mathbf{k}, \omega)$. (b) For $T \sim T_{f}$, the quasiparticle band is interrupted by the incoherent $4 f$ electrons. The red dotted line is provided as a guide for the high $T$ band. (c) For $T_{f}>$ $T>T_{m}$, the hybridization between non- $f$ and $4 f$ electrons is strengthened. (d) For $T<T_{m}$, the HG and $k$-dependent (dispersive) KR state can be well defined. The orange color represents $A^{f}(\mathbf{k}, \omega)$. With lowering $T, E_{F}$ increases gradually due to the participation of the new carrier from the incoherent $4 f$ electrons. The FS on the $\mathbf{k}=Y$ plane at high and low $T$ are provided in (e) and (f), respectively. Color represents the different band indices. The scaling behaviors of the $T$-dependent renormalized de Haas van Alphen frequency $F$ and cyclotron effective mass $m^{*}$ for the FS branch $\alpha$ shown in (e) and (f) are analyzed in (g) and (h), respectively.

$\left(T_{m}\right)$ for the $T$-dependent evolution of the cyclotron effective mass $m^{*} . T_{f}$ should be related to the contribution of the local $4 f$ electron to the conduction electron. On the other hand, $T_{m}$ is a characteristic of the formation of coherent $4 f$ bands in the lattice since the $m^{*}$ reflects the renormalization of the carriers. Although $T_{m}$ is defined by the change of the effective mass of the FS, it should be similar to $T^{*}$ where the the formation of the coherent KR states begins. ${ }^{31}$ Similar to CeIrIn 5 , the same scaling behavior is also shown in $\mathrm{CeCoGe}_{2}$. The FS branch $\alpha$, which is the well-defined FS branch at all $T$, as shown in Figs. 4(e) and 4(f), is chosen for this study. By analyzing $T$-dependent scaling behaviors in Figs. 4(g) and 4(h), we found $T_{f} \sim 200$ and $T_{m} \sim 90 \mathrm{~K}$, respectively, for $\alpha$ branch.

The kink can be observed around $T_{f}$, where the incoherent $f$ state contributes to $E_{F}$. (See the Supplemental Material. ${ }^{32}$ ) The kink phenomenon is changed into the gap feature gradually between $T_{f}$ and $T_{m}$, where the contribution of incoherent $4 f$ electron states disturb the band dispersion near $E_{F}$. Well below $T_{m}$, the HG and KR states are well defined. So, the formations of the kink around $T_{f}(200 \mathrm{~K})$ will be the precursor of the HG below $T_{m}(90 \mathrm{~K})$. Note that the kink features are also observed around $0.3 \mathrm{eV}$ above $E_{F}$, as shown in Fig 3(d), due to the incoherent contribution of the spin-orbit multiplet. The multiplet around $-0.3 \mathrm{eV}$ does not give the kink because the contribution of the $f$ state is too weak to distort the non- $f$ bands.
In summary, we have analyzed the $T$-dependent evolutions of $A(\mathbf{k}, \omega)$ in the heavy fermion compound $\mathrm{CeCoGe}_{2}$. It is shown that the DFT + DMFT calculations are consistent with the experimental measurements. We propose that the kink of $A^{\text {non- } f}(\mathbf{k}, \omega)$ around $E_{F}$ can be identified during the evolution from the dispersive non- $f$ state at high $T$ to the HG and KR states at low $T$. Phenomenologically, the kinks observed in this work will show the similar shape to those in other experiments, even though the conventional kinks appear in the correlated bands via the interaction with other excitations, such as phonon and spin fluctuations. The kinks in the current work should be distinguished also from the one only with the electronic correlation in previous studies. ${ }^{22-24}$ As indicated in Fig. 4, the kink can be observed between $T_{m}$ and $T_{f}$, while all other kinks in previous studies should be shown well below $T^{*}\left(\sim T_{m}\right)$. The kink induced by the correlation between the incoherent $4 f$ and dispersive non- $f$ electrons above $T^{*}$ can be investigated in the state-of-the-art $T$-dependent ARPES experiments. We suggest that the detailed analysis on the abrupt change of electron velocity and the scattering rate near the Fermi level will provide crucial information for the heavy fermion system.

We acknowledge useful discussions with Hojun Im (Hirosaki University) and Tuson Park (Sungkyunkwan University). This work was supported by the National 
Research Foundation of Korea (NRF) and funded by the Ministry of Education, Science, and Technology (Grants No.
2009-007994, No. 2010-0006484, No. 2010-0026762, and No. 2012029709). *bimin@ postech.ac.kr

†jhshim@postech.ac.kr

${ }^{1} \mathrm{P}$. Coleman, in Lectures on the Physics of Highly Correlated Electron Systems VI: Sixth Training Course in the Physics of Correlated Electron Systems and High-Tc Superconductors, AIP Conf. Proc. No. 629 (AIP, Melville, NY, 2002), pp. 79-160.

${ }^{2}$ J. W. Allen, J. Phys. Soc. Jpn. 74, 34 (2005).

${ }^{3}$ H. J. Im, T. Ito, H-D. Kim, S. Kimura, K. E. Lee, J. B. Hong, Y. S. Kwon, A. Yasui, and H. Yamagami, Phys. Rev. Lett. 100, 176402 (2008).

${ }^{4}$ M. Klein, A. Nuber, H. Schwab, C. Albers, N. Tobita, M. Higashiguchi, J. Jiang, S. Fukuda, K. Tanaka, K. Shimada, M. Mulazzi, F. F. Assaad, and F. Reinert, Phys. Rev. Lett. 106, 186407 (2011).

${ }^{5}$ F. Marabelli and P. Wachter, Phys. Scr. T 45, 120 (1992).

${ }^{6}$ S. V. Dordevic, D. N. Basov, N. R. Dilley, E. D. Bauer, and M. B. Maple, Phys. Rev. Lett. 86, 684 (2001).

${ }^{7}$ C. I. Lee, K. E. Lee, Y. Y. Song, H. J. Im, S. Kimura, and Y. S. Kwon, Infrared Phys. Technol. 51, 488 (2008).

${ }^{8}$ Y. S. Kwon, K. E. Lee, M. A. Jung, E. Y. Song, H. J. Oh, H. J. Im, and S. Kimura, J. Magn. Magn. Mater. 310, 310 (2007).

${ }^{9}$ S. Donovan, A. Schwartz, and G. Grüner, Phys. Rev. Lett. 79, 1401 (1997).

${ }^{10}$ D. A. Bonn, J. D. Garrett, and T. Timusk, Phys. Rev. Lett. 61, 1305 (1988).

${ }^{11}$ J. H. Shim, K. Haule, and G. Kotliar, Science 318, 1615 (2007).

${ }^{12}$ E. D. Mun, B. K. Lee, Y. S. Kwon, and M. H. Jung, Phys. Rev. B 69, 085113 (2004).

${ }^{13}$ A. Lanzara, P. V. Bogdanov, X. J. Zhou, S. A. Kellar, D. L. Feng, E. D. Lu, T. Yoshida, H. Eisaki, A. Fujimori, K. Kishio, J.-I. Shimoyama, T. Noda, S. Uchida, Z. Hussain, and Z.-X. Shen, Nature (London) 412, 501 (2001).

${ }^{14}$ G.-H. Gweon, T. Sasagawa, S. Y. Zhou, J. Graf, H. Takagi, D.-H. Lee, and A. Lanzara, Nature (London) 430, 187 (2004).

${ }^{15}$ E. Schachinger, J. P. Carbotte, and T. Timusk, Europhys. Lett. 86, 67003 (2009).
${ }^{16}$ T. Dahm, V. Hinkov, S. V. Borisenko, A. A. Kordyuk, V. B. Zabolotnyy, J. Fink, B. Büchner, D. J. Scalapino, W. Hanke, and B. Keimer, Nat. Phys. 5, 217 (2009).

${ }^{17}$ J. Hwang, T. Timusk, and G. D. Gu, Nature (London) 427, 714 (2004).

${ }^{18}$ M. Hengsberger, D. Purdie, P. Segovia, M. Garnier, and Y. Baer, Phys. Rev. Lett. 83, 592 (1999).

${ }^{19}$ T. Valla, A. V. Fedorov, P. D. Johnson, and S. L. Hulbert, Phys. Rev. Lett. 83, 2085 (1999).

${ }^{20}$ Eli Rotenberg, J. Schaefer, and S. D. Kevan, Phys. Rev. Lett. 84, 2925 (2000).

${ }^{21}$ T. Durakiewicz, P. S. Riseborough, C. G. Olson, J. J. Joyce, E. Bauer, J. L. Sarrao, S. Elgazzar, P. M. Oppeneer, E. Guziewicz, D. P. Moore, M. T. Butterfield, and K. S. Graham, Europhys. Lett. 84, 37003 (2008).

${ }^{22}$ K. Byczuk, M. Kollar, K. Held, Y.-F. Yang, I. A. Nekraov, Th. Pruschke, and D. Vollhardt, Nat. Phys. 3, 168 (2007).

${ }^{23}$ A. Kainz, A. Toschi, R. Peters, and K. Held, Phys. Rev. B 86, 195110 (2012).

${ }^{24}$ K. Held, R. Peters, and A. Toschi, Phys. Rev. Lett. 110, 246402 (2013).

${ }^{25}$ A. Macridin, M. Jarrell, T. Maier, and D. J. Scalapino, Phys. Rev. Lett. 99, 237001 (2007).

${ }^{26}$ Shiladitya Chakraborty, Dimitrios Galanakis, and Philip Phillips, Phys. Rev. B 78, 212504 (2008).

${ }^{27}$ C. Raas, P. Grete, and G. S. Uhrig, Phys. Rev. Lett. 102, 076406 (2009).

${ }^{28}$ G. Kotliar, S. Y. Savrasov, K. Haule, V. S. Oudovenko, O. Parcollet, and C. A. Marianetti, Rev. Mod. Phys. 78, 865 (2006).

${ }^{29}$ S. Y. Savrasov, Phys. Rev. B 54, 16470 (1996).

${ }^{30} \mathrm{R}$. D. Cowan, The Theory of Atomic Structure and Spectra (Univ. California Press, Berkeley, 1981).

${ }^{31}$ Hong Chul Choi, B. I. Min, J. H. Shim, K. Haule, and G. Kotliar, Phys. Rev. Lett. 108, 016402 (2012).

${ }^{32}$ See Supplemental Material at http://link.aps.org/supplemental/ 10.1103/PhysRevB.88.125111 for the $T$-dependent variation of the non- $f$ spectral function $A(\mathbf{k}, \omega)$.

${ }^{33}$ The structure is drawn by the VESTA package. K. Momma and F. Izumi, J. Appl. Crystallogr. 41, 653 (2008). 